\title{
Special issue on robotics: science and systems
}

\author{
Gregory Dudek · Dieter Fox
}

Published online: 30 September 2014

(C) Springer Science+Business Media New York 2014

This issue of Autonomous Robots presents journal articles that are based on papers originally presented at the 2013 Robotics Science and Systems conference, held in Berlin, Germany. Although these were selected by a committee to exemplify the best papers presented that year, the decision over which papers to include was a difficult one due to the substantial number of very strong papers in the cohort. While the strength of these papers can be partially attributed to the fact that the conference itself generally attracts strong papers, it is also a sign of the maturation of our field. The papers in this issue reflect a few broad themes that are currently attracting the attention of the robotics research community: the development of multi-agent systems (where the agents can be either multiple robots, or a mixture of humans and robots), the link between sensing and manipulation in robotic systems, and the continuing use of sophisticated probabilistic representations in robot control.

In the paper "Multivariate Evaluation of Interactive Robot Systems" a method for evaluating interactive robotic systems is described. This work emphasizes the ability to evaluate a complex system with a large number of design variables using tools from multiple regression, and does so in the context of human-robot interaction where exhaustive methods would be impractical. The context is that of understanding

\section{G. Dudek ( $\varangle)$}

School of Computer Science, McGill University, 3480 University

Street, Montreal, QC H3A 2A7, Canada

e-mail: dudek@cim.mcgill.edu

URL: http://www.cim.mcgill.ca/ dudek

D. Fox

Paul G. Allen Center for Computer Science and Engineering, University of Washington, 185 Stevens Way, Seattle,

WA 98105 , USA

e-mail: remove@mail.com

URL: http://www.homes.cs.washington.edu/ fox how expressive gestures made by a robot will impact the subjective experience of a human listener.

The role of humans in robotics deployments is illustrated again in the paper "Integrating Human Observer Inferences into Robot Motion Planning." This work explicitly takes into account the role of a human that may be observing a robot's activities to maximize the extent to which the robot's intentions are apparent. This paper introduces and formalizes the notion of legible motion, which is to say actions for which intentionality is readily apparent, and tests the notion experimentally.

The relationship between effective grasping and manipulation of physical objects, and our ability to sense these properties is explored in the paper "Perceiving, Learning, and Exploiting Object Affordances for Autonomous Pile Manipulation," in which feasible manipulation strategies are computed using an RGB-D image and depth sensor. The paper takes this algorithmic notion to its fullest realization by describing a system in which a robot can be directed to completely clear a cluttered table by individually grasping and removing each object. The effectiveness of the approach is demonstrated by extensive experimental data comparing the results to both human performance and alternative algorithmic formulations.

The paper "Approximate Representations for Multi-Robot Control Policies that Maximize Mutual Information" addresses issues at the intersection of optimal control, probabilistic modeling, and active sensing. In it, the authors address the problem of controlling a small team of robots to estimate the location of a mobile target using simple non-linear range-only sensors. A goal of the resulting trajectory control is to produce trajectories that will maximize the mutual information between the future state of the target robot and the measurements. The key contribution is a new approximation to the problem and an associated theo- 
retical design criterion that allows the controller to be formulated.

The paper "Goal Assignment and Trajectory Planning for Large Teams of Interchangeable Robots" also deals with multi-robot trajectory planning, and focuses on theoretical constraints related to optimality, but from a very different perspective. The emphasis is on large teams and optimization through interchanging the goals of the individual robots. The key aspect of the work is to produce a tractable algorithm for this demanding problem, a theoretical complexity bound, and then an analysis of its performance in practice.

The theme of algorithm design for large collections of robots is continued in the paper "From Selfish Auctioning to Incentivized Marketing," in which a much broader notion of robot objectives is considered, including context where there may be a mixed combination of objectives. The paper presents an elegant action-based approach where tasks are represented as goods in an auction to assure that all tasks are assigned and that a state of equilibrium is achieved, as represented by an optimum for the parties involved in the process. The approach allows for a distributed implementation.

This set of six papers provides an excellent benchmark for the flavor of the Robotics Science and Systems conference, as well as an illustration of the interplay between formal analysis and systems design in the context of robotics today. 